\title{
Is There Mother-Infant Bonding in Primates?
}

\author{
Dario Maestripieri
}

\section{Committee on Human Development, University of Chicago, and Yerkes Regional Primate Research Center, Emory University}

\begin{abstract}
The study of mother-infant bonding was stimulated by concepts and data imported from animal research. However, the occurrence of mother-infant bonding in nonhuman primates has not been systematically investigated. Information obtained from naturalistic observations of infant adoption, experimental studies of infant cross-fostering, and studies of mother-infant recognition in primates suggest that the first few days of the postpartum period are a sensitive period for maternal motivation. During this period, primate mothers are highly attracted to infants and motivated to take care of them, and therefore in optimal conditions to bond with their offspring. This does not necessarily imply, however, that bonding cannot occur during subsequent postnatal periods or that small differences in time spent in contact by mother and infant during the early postpartum days have long-lasting consequences for development or parenting. The determinants of temporal changes in maternal motivation during the postpartum period or of individual differences in maternal motivation have been poorly investigated in humans. The primate evidence can stimulate human research in this area and enhance our understanding of some adaptive and maladaptive processes underlying parenting behavior and child development. (c) 2001 Academic Press
\end{abstract}

In a popular book published in the mid 1970s, Klaus and Kennell (1976) hypothesized that, similar to what occurs in some animals, there exists in humans a brief period immediately after birth during which mother and infant develop a strong, affectionate bond. In support of their hypothesis, they provided some evidence indicating that additional contact between mother and infant during this period had long-lasting positive effects for parenting and child development whereas separation could result in negative consequences, including perhaps child abuse and neglect. The Klaus and Kennell idea stim-

I thank Scott Lilienfeld for comments on the manuscript. This research was supported by a grant from the L.S.B. Leakey Foundation, NIMH Grants R01-MH57249 and R01-MH62577, and in part, by NIH Grant RR-00165 to the Yerkes Regional Primate Research Center. The Yerkes Center is fully accredited by the American Association for Accreditation of Laboratory Animal Care.

Address reprint requests to Dario Maestripieri, Committee on Human Development, University of Chicago, 5730 S. Woodlawn Avenue, Chicago, IL 60637. Fax: (773) 702-0320. Email: dario@ccp.uchicago.edu.

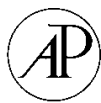


ulated a great deal of research in subsequent years, some of which provided evidence in support of the bonding hypothesis. Such research, however, was later criticized on both scientific and ideological grounds (Eyer, 1994; Goldberg, 1983; Lamb \& Hwang, 1982; Myers, 1984; Siegel, 1982; Sluckin \& Sluckin, 1982). Most of the scientific criticism focused on the methodological flaws of bonding studies and concluded that any claims concerning the developmental effects of brief periods of mother-infant contact or separation after parturition were not warranted. Thus, many reviewers concluded that the available evidence did not support the notion of a critical or sensitive period for mother-infant bonding.

Unfortunately, as pointed out by Lamb and Hwang (1982) the concepts of "critical" and "sensitive" periods have often been confused by some of the bonding researchers and their critics. The notion of critical period refers to a limited period during development during which specific events must occur to ensure normal development. The same events occurring before or after the critical period will have little or no effect. The notion of critical period originated from early animal research where it was used, for example, to describe the developmental process through which young birds become "imprinted"' on their parents (Lorenz, 1937). Many researchers, however, subsequently argued that true critical periods do not occur in animals because the beginning and ending of such periods are more flexible than previously thought and because events occurring during the critical periods that were believed to be irreversible were in fact partly or totally reversible (Bateson, 1966, 1979; Salzen \& Meyer, 1968). Animal researchers have therefore replaced the notion of critical periods with that of sensitive periods, by which they mean periods of time with relatively flexible boundaries in which developing organisms are most responsive to certain environmental stimuli and most likely to be affected by them (Bateson, 1979).

If true critical periods do not occur in animals, they are even less likely to occur in humans, as human development and behavior are particularly plastic and complex. In fact, in the revised edition of their book, Klaus and Kennell (1982) emphasized that the human data were not consistent with the notion of a critical period. Rather, they suggested that the first postpartum days could be considered a "maternal sensitive period" in that, during this period, mothers may be especially responsive to their babies so that contact with the baby during this period is likely to enhance the mother-child relationship (see also Kennell \& Klaus, 1984). Several critics of their research, however, rejected also this softer version of the bonding hypothesis (e.g. Myers, 1984). In fact, some authors such as Lamb and Hwang (1982) concluded that maternal sensitive periods in mammals are an exception limited to ungulates (e.g., sheep and goats) and that parental behavior in primates and humans is so complex and flexible that physiological influences or sensitive periods are unlikely to occur (see also Sluckin \& Sluckin, 1982). Their con- 
clusion, however, was at least in part based on the lack of relevant research rather than on research producing negative results.

In the early 1980s, when most of the critical reviews of the bonding literature were published, evidence of physiological regulation of maternal behavior in mammals had been reported only for rats and sheep (Numan, 1994) and a sensitive period for mother-offspring bonding had been documented only in ungulates. An early study of sheep and goats had shown that newborn kids and lambs reunited with their mother after a separation shorter than 45 min were accepted, whereas those separated for longer than 45 min were rejected (Collias, 1956). Subsequent studies showed that 5-10 min of contact after birth were sufficient for the mother to recognize her offspring's odor and prevent rejection after separation and reunion (Herscher, Moore, \& Richmond, 1958; Herscher, Richmond, \& Moore, 1963; Klopfer, 1971; Klopfer, Adams, \& Klopfer, 1964). Mothers, however, could be induced to adopt alien young that were exchanged with their offspring provided that these young had not been for too long with another mother (Herscher et al., 1963; Klopfer, 1971; Gubernick, 1980). These findings suggested that there is a brief sensitive period after birth (whose exact length remained unclear; Gubernick, 1980) during which mothers identify and bond with their offspring. Maternal responsiveness and olfactory recognition of the offspring in the immediate postpartum period have been shown to be linked to some of the physical and neuroendocrine events of the periparturitional period (Keverne \& Kendrick, 1990).

At the time that most critical reviews of the bonding literature were published, there had been few or no studies of neuroendocrine influences on maternal behavior in primates or humans. Over the past few years, however, possible endocrine influences on maternal responsiveness and behavior in monkeys and humans have begun to be systematically investigated (Maestripieri, 1999, in press). For example, a recent study of macaques showed that maternal responsiveness increased during late pregnancy and after estrogen treatment (Maestripieri \& Zehr, 1998). Furthermore, Fleming, Ruble, Krieger, and Wong (1997) showed that human mothers who maintained high levels of estradiol over the parturitional period also had higher feelings of attachment to their own infant in the early postpartum days than mothers whose estradiol levels dropped. Thus, in contrast to earlier suggestions, these studies indicate that primate and human parenting are not completely emancipated from physiological variables.

In the last few years, a great deal of research has also been conducted on early mother-infant interactions in primates, and some of this research has produced information potentially useful to the investigation of maternal sensitive periods. This information, however, has not been critically reviewed or explicitly discussed in relation to mother-infant bonding. In fact, some of this information has been provided by infant cross-fostering studies that 
were conducted for practical rather than scientific purposes. Information relevant to mother-infant bonding has also been made available by naturalistic observations of infant adoption in free-ranging monkeys and studies of mother-infant recognition through visual or vocal stimuli.

In light of the new information that has been made available by animal research in the past 15-20 years (e.g., Colgan, 1983; Hepper, 1991; Maestripieri \& Call, 1996), it may be worth reassessing the claim that a sensitive period for mother-infant bonding is a mammalian exception limited to ungulates. Specifically, in this article I will address the question of whether a maternal sensitive period occurs in primates as well. In doing so, I will not attempt to demonstrate what most human research on bonding has attempted to demonstrate, i.e., that different amounts of mother-infant contact during the postpartum period have long-lasting quantitative and qualitative consequences for the mother-offspring relationship. In fact, to my knowledge, these effects have not been unequivocally demonstrated in any animal species, including ungulates. Rather, I will investigate whether the phenomena of adoption of alien young and offspring rejection following motheroffspring separation that have been observed in ungulates also occur in primates.

Based on the evidence from ungulates, my working hypothesis in this investigation is that a gradual process of selective bonding to one infant begins at parturition and is completed at some point during the early postpartum period. Early on, this period of bonding formation is characterized by enhanced responsiveness to all infant stimuli and low threshold for discrimination between familiar and unfamiliar infants. Later on, a gradual reduction in responsiveness to all infant stimuli is accompanied by increasing familiarity with and recognition of the characteristics of the mother's own offspring. My working hypothesis leads to the following three predictions: (1) when mother and infant are separated during the bonding period, the mother will accept her own infant or an alien infant with similar characteristics if reunion occurs before the end of the bonding period; (2) when mother and infant are separated during the bonding period, the mother will reject her own infant and any other infant if reunion occurs after the end of the bonding period; (3) when mother and infant are separated after the bonding period and later reunited, the mother will accept her own infant but reject any other infant.

To gather information relevant to mother-infant bonding in primates, I will review the evidence provided by naturalistic observations of infant adoption, experimental studies of infant cross-fostering, and studies of motherinfant recognition. Most of the relevant information comes from monkeys of the genus Macaca because early mother-infant interactions have been studied in macaques more than in other primates, particularly in the laboratory. Information concerning other species of monkeys or apes, however, will be reviewed and discussed whenever available. The article will be concluded by a discussion section in which I assess whether the predictions of 
my bonding hypothesis are supported by the primate data, discuss the possible mechanisms underlying mother-infant bonding in primates, and finally interpret the bonding phenomenon from a comparative and evolutionary perspective with some implications for human research.

\section{THE EVIDENCE}

\section{Naturalistic Observations of Infant Adoption}

For the purpose of this review, infant adoption is defined as a permanent association between a lactating female and a nutritionally dependent infant other than her offspring, which is initiated during the postpartum period and in which the female shows all the patterns of maternal behavior typically shown with offspring (e.g., suckling, cradling, retrieving) (see Thierry \& Anderson, 1986, for a broader review of different adoption phenomena).

Naturalistic observations of infant adoption in group-living primates have been made in two main contexts. One situation involves mothers whose infant dies soon after birth and adopt another newborn. The second situation involves females with live offspring who adopt an additional newborn and raise it along with their biological offspring. The adopted infant has usually been abandoned by another parturient female or in some cases forcibly kidnapped from her.

Lactating females whose infants die typically show increased interest in other females' infants. For example, in pigtail macaques (Macaca nemestrina) lactating females who lost their infant in the first or sixth week postpartum showed significantly higher frequencies of interactions with other females' infants than lactating females who did not lose their infant (Maestripieri \& Wallen, 1995). Two weeks after infant loss, the frequencies of infant-directed behavior of lactating females with and without infants were no longer significantly different. Interactions with other females' infants may range from brief handling to kidnapping attempts. Whether or not females who lose their offspring adopt another infant depends on the opportunities available for adoption. At the Yerkes Primate Center, one rhesus macaque (Macaca mulatta) female who had a stillbirth permanently adopted an infant born the following day that had been abandoned by its mother (personal observation). In the colony of Japanese macaques (Macaca fuscata) of the Rome Zoo in Italy, one primiparous mother who lost her female infant 2 days after parturition kidnapped and adopted a 6-week-old female infant 8 days later (Schino et al., 1993). Finally, one green monkey (Cercopithecus sabaeus) mother living in a captive group on La Cueva Island, Puerto Rico, who lost her 3-week-old male infant kidnapped and adopted another 2-weekold male infant (Marsden \& Vessey, 1968). The foster mother had shown interest in the younger infant since its birth but the death of her own offspring triggered the adoption process.

Adoption of an additional infant without offspring loss has been reported 
in both Japanese and rhesus macaques. In captive Japanese macaques, Fuccillo, Scucchi, Troisi, and D'Amato (1983) reported the case of a mother with a 2-day-old female infant who adopted another female newborn that had been abandoned. In free-ranging Japanese macaques, Ogawa (1998) reported the case of two mothers with male infants who adopted another newborn male infant the day after parturition. In both cases, the adopted infants had been rejected and abandoned by their mothers. Among rhesus macaques housed at the California Primate Center, Ellesworth and Andersen (1997) observed four mothers who adopted and raised an additional infant. Three mothers were multiparous and one primiparous. The ages of the biological infants at adoption were 2, 5, 15, and 15 days. The ages of the adopted infants at adoption were $1,1,2$, and 10 days. Three offspring were male and one female, while all the four adopted infants were male. In another case of multiple adoption observed at the Yerkes Primate Center, one rhesus female and her adult daughter gave birth on two consecutive days and each adopted another infant the day following parturition (personal observation, April 1993). The older mother was multiparous while her daughter was at her first reproductive experience. Three infants were female and one male. The origin of the two adopted infants remained unknown. Finally, Clarke and Glander (1981) reported the case of a free-ranging howler monkey (Alouatta palliata) infant, whose sex was unknown and age was estimated to be 4-6 months, who after losing its mother was often observed near another lactating female and was presumed to be adopted by her.

The above reviewed naturalistic observations of infant adoption share some interesting features. First, with one exception, all reported cases of single or multiple adoption took place within the first 2-3 postpartum weeks. Second, with a few exceptions, in most cases of infant adoption associated with offspring loss, the infants that were adopted were of the same sex and similar age as the offspring. Thus, although the occurrence of adoption is, to some extent, constrained by the availability of infants to adopt, mothers may play a more active role in the selection of adopted infants than previously thought.

It may be argued that the naturalistic observations of infant adoption, particularly in the wild, are too few for this phenomenon to have any biological significance. It must be emphasized, however, that infant adoption is probably largely underreported. In fact, in many cases, human observers have no way of knowing whether the infant they see with a new mother was the same as the one they saw the day before (e.g., see Bernstein, 1991). The fact that the circumstances in which infant adoption occurs in the wild can be successfully reproduced in the laboratory suggests that primate females have the potential to adopt unrelated infants. For example, cases in which newly parturient females are induced to "replace" their dead offspring or to adopt an additional infant are known for many species of primates (e.g., squirrel monkeys) (Saimiri sciureus), Eveleigh \& Hudson, 1973; Taub, Lehner, \& Adams, 
1977; common marmosets (Callithrix jacchus), Box, 1984; long-tailed macaques (Macaca fascicularis), Timmermans, Schouten, \& Krijnen, 1981; stump-tailed macaques (Macaca arctoides), Chamove \& Anderson, 1982; chimpanzees (Pan troglodytes), Van Wulfften Palthe \& van Hooff, 1975; lowland gorillas, Nakamichi, 1998).

\section{Experimental Studies of Infant Cross-Fostering}

Infant cross-fostering, i.e., the exchange of infants between two lactating females, is one special case of infant adoption. Infant cross-fostering not induced by human manipulations is a very rare event among group-living monkeys or apes. One such rare event involved two female olive baboons (Papio cynocephalus) living in captivity (Lawrence, 1982). At the time that the exchange of infants was discovered, the infants were 18 and 29 days old, respectively; one was male and the other female. It was not clear when the transfer occurred, but one of the two mothers must have been at least in her second week postpartum. The mother who adopted the younger infant later during her postpartum period subsequently rejected this infant whereas the mother who adopted the older infant earlier during her postpartum period successfully raised this infant.

Although "'spontaneous"' infant cross-fostering is rare, experimental procedures in which infants are swapped between two lactating females have been carried out in many primate research and breeding facilities for a variety of reasons. For example, Smith (1986) proposed infant cross-fostering as a procedure to increase genetic diversity within breeding groups of rhesus macaques. Champoux, Boyce, and Suomi (1995) cross-fostered rhesus macaque infants to investigate environmental influences on bio-behavioral development. Owren and Dieter (1989) cross-fostered infants belonging to two different macaque species, the rhesus and the Japanese macaque, to investigate biological and learning components of vocal development. Finally in our laboratory we recently cross-fostered rhesus infants to investigate biological and learning components of maternal abusive behavior (Maestripieri, Megna, \& Jovanovic, 2000).

Interestingly, when providing a rationale for their cross-fostering procedures, several authors used arguments implying the occurrence of a sensitive period for mother-infant bonding. For example, Smith (1986) stated that "infants less than two weeks old were preferred for cross-fostering because there is evidence that at this age mothers have not yet established a strong affectional bond with their infants"' (p. 231). Owren and Dieter (1989) believed that "a fostering was deemed most likely to succeed if the biological and foster infants were less than 2 weeks old, if they were age-matched to within a few days, and if the separation interval during which the prospective foster mother was deprived of any infant contact was minimized"' (p. 246). Finally, Champoux et al. (1995) reported that "it was necessary to effect the adoption by the third postpartum day, in order to ensure the viability of 
lactation and to prevent the mother's attachment to her biological infant', (p. 7). Thus, some researchers hint at the occurrence of mother-infant bonding in primates although they appear to rely on common sense knowledge rather than on solid empirical evidence.

To investigate whether these claims are supported by empirical evidence, I analyzed a large set of infant cross-fostering data obtained from the following studies: Smith (1986), Holman and Goy (1988), Owren and Dieter (1989), and Maestripieri et al. (2000). Data provided by these studies could be reanalyzed because these studies shared the following features: (1) mothers and infants lived in social groups rather than in individual cages; (2) mothers were born either in the wild or in captivity but had been raised by their own biological mothers rather than by humans; (3) the cross-fostering procedure consisted of temporarily removing two mother-infant pairs from their groups, placing mothers in standard squeeze cages, and switching infants between them; (4) the adoption attempt lasted a few minutes or hours and was immediately terminated if the infant was violently rejected (e.g., slapped or bitten) by the foster mother; no forced cohabitation lasting several days was imposed on mothers and rejected infants. The Maestripieri et al. (2000) cross-fostering study also involved some abusive mothers but these individuals were not included in the present analysis.

Cross-fostering data were analyzed to investigate whether the successful adoption of a foster infant was predicted by variables such as the mother's age, the number of her previous offspring that survived at least to 1 year of age, the time elapsed since parturition, the duration of separation, and the differences in age and sex, if any, between biological offspring and foster infant. The total dataset, shown in Table 1, consists of 61 adoption attempts, 48 of which were successful and 13 unsuccessful. Three animals were sampled twice and the outcome of the adoption was the same in both cases (1 positive and 2 negative). Information on the number of previous offspring was not available for the subjects in Owren and Dieter's (1989) study. Three subjects in Smith's (1986) study had stillbirth, and information on the time elapsed between parturition and the cross-fostering attempt was not available for them. For these 3 subjects, separation time was arbitrarily set at $12 \mathrm{~h}$, considering that many rhesus mothers deliver their infants at night or on weekends and their stillborns are often not discovered until the day following parturition (personal observations).

The cases of successful adoption did not differ from those of rejection in terms of the mother's age (Mann-Whitney test, $z=-.89$; NS), the number of previous offspring $(z=-.19$; NS), the time elapsed since parturition $(z=-.73, \mathrm{NS})$, the age difference between biological and foster infant $(z=-1.05, \mathrm{NS})$, or whether the sex of the biological and foster infants was the same or different $\left(\chi^{2}=1.91, \mathrm{NS}\right)$. However, the duration of separation was significantly lower for the successful adoptions than for the unsuccessful ones $(z=-2.93 ; \mathrm{p}<.01)$. The difference was statistically significant even 
if the 3 mothers with stillborns $(18383,17664$, and 7047) and the two mothers with the highest values of separation time (22089 and RFr2) were excluded from the analysis $(z=-2.12 ; \mathrm{p}<.05)$. Moreover, the mothers with separation duration of $1 \mathrm{~min}$ were significantly more likely to adopt infants (25 of 27) than the mothers with separation duration longer than 1 min (23 of $\left.34 ; \chi^{2}=5.58, p=.01\right)$.

\section{Other Cross-Fostering Studies}

Studies reviewed in this section were characterized by the use of individually caged animals, some of which had an unknown or abnormal developmental history, rather than group-living animals. Since individual housing or abnormal developmental history can affect mother-infant interactions in primates (e.g., Meier, 1965; Maestripieri \& Carroll, 1998) and hence potentially also the outcome of the cross-fostering experiment, these studies are reviewed and discussed separately. Studies involving a long period of forced cohabitation between mothers and foster infants despite repeated infant rejection are also reviewed separately. This is because cases of adoption in which the mother accepted the foster infant after days of forced cohabitation would have been considered unsuccessful in other studies that did not use such a stressful procedure. The findings of studies involving forced cohabitation are difficult to interpret and will not be used as evidence for or against the bonding hypothesis.

In one of the earliest studies of infant cross-fostering, Jensen and Tolman (1962) briefly separated two individually caged pigtail macaque mothers from their infants, one of which was 5 months old and the other 7 months old. The mothers were then reunited either with their own infant or with the other one. Both mothers showed recognition and acceptance of their own infant and indifference and rejection toward the strange infant, thus demonstrating that adoptions are unlikely to be successful when infants are several months old. Deets and Harlow (1974) subsequently reported some cases of successful adoption by individually caged rhesus macaque females. Eight multiparous mothers were separated from their infants at birth; four of them were offered one infant for adoption and the other four were offered two infants. Cross-fostering was conducted between 1 and 12 days following parturition (on average 2.5 days) and the age of foster infants ranged from 1 to 9 days. All four attempts at single adoption were successful whereas three of four mothers with twins rejected one of their foster infants. All infants in this study, however, were left in a cage with their foster mothers for several hours or days even if they had been violently rejected by them.

A more recent study that also used individually caged adult females and involved forced cohabitation between mothers and foster infants was conducted with longtail macaques (Macaca fascicularis) by Cho, Suzuki, and Honjo (1986). In this study different types of infant adoption procedures were attempted with 122 mothers. One group of subjects consisted of 20 


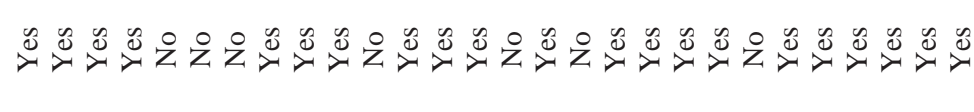

$-m$ กำ 岂.

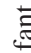

플

!

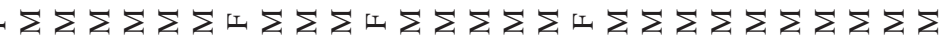

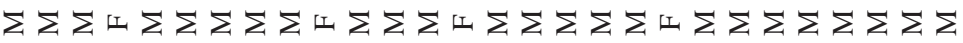

구우의

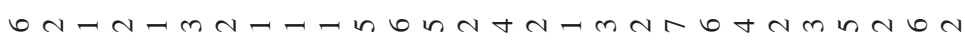

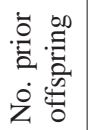

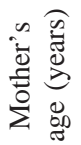

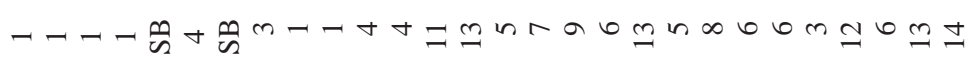

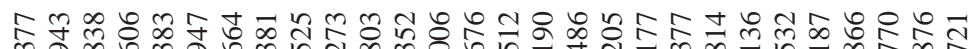

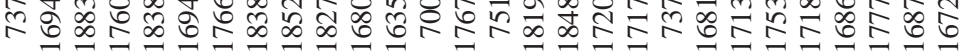

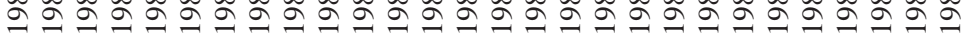

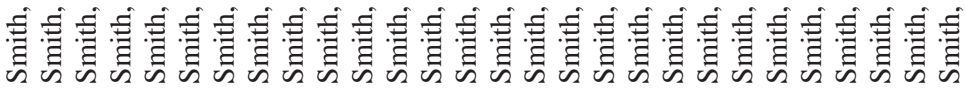




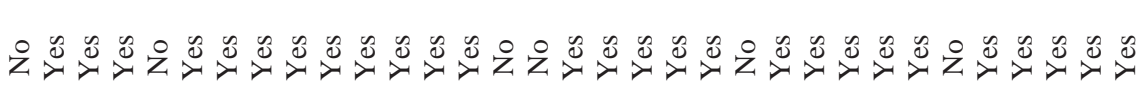

斻-

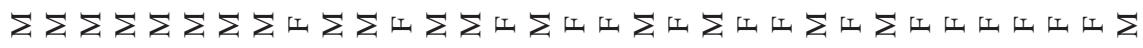

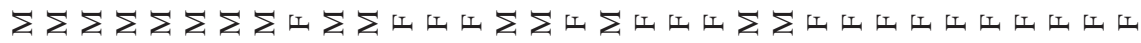

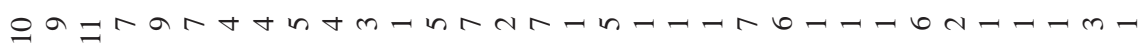

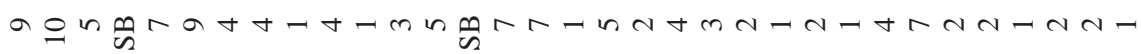

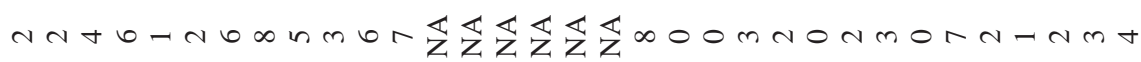

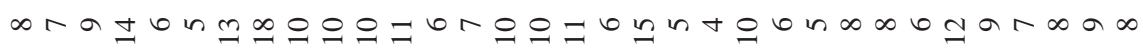

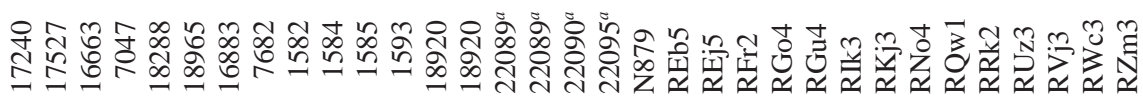
œ

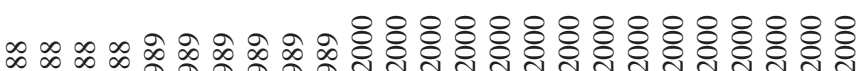

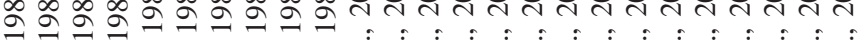

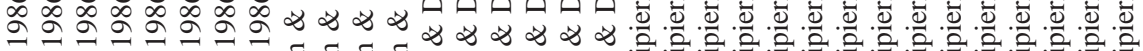

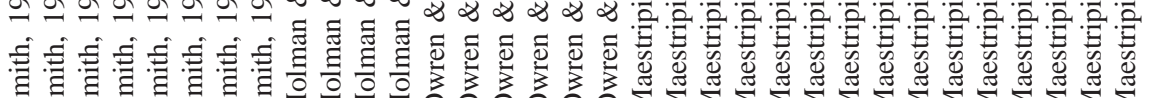

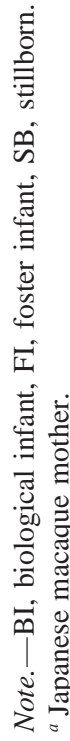


mothers who had a stillbirth and were given a foster infant (age, 0-28 days; mean $=4.7 \pm 6.8$ ) $0-4$ days after parturition. Fifteen adoptions were successful and 5 failed. In a second group of 21 subjects, mothers were separated from their infants after 3 months and given a foster infant (age, 0-56 days; mean $=9.6 \pm 17.9) 0-4$ days after separation. Ten mothers adopted the foster infants and 11 rejected them. In a third adoption procedure, 4 mothers were separated from their infants at ages $0,1,1$, and 3 and given foster infants of ages 2, 1, 0, and 8. All adoptions were successful. Finally, 77 mothers were separated from their infants of ages $0-24$ days $(1.1 \pm 3.5)$ and given two infants, one of which was their own offspring and the other an unrelated infant (age, $0-39$ days; mean $=2.3 \pm 5.8$ ). The difference in age between own offspring and foster infant was 0-39 days $(2.5 \pm 6.0)$. In this group of subjects, 57 adoptions succeeded and 20 failed. Sex of the foster infant and number of days since separation did not account for adoption success in any of the four experimental groups, although the authors acknowledged that the information on these two variables was not always accurate. It also needs to be emphasized that all adoption attempts, whether successful or unsuccessful, involved forced cohabitation of mothers and foster infants for at least 7 days.

A related study conducted with the same species used a different crossfostering procedure to investigate how early during the postpartum period mothers discriminated between their own and foster infants (Negayama \& Honjo, 1986). Sixty individually caged mother-infant pairs served as subjects in tests in which mothers were first separated from their own infants for $5 \mathrm{~min}$, then given a foster infant for $5 \mathrm{~min}$, and then reunited with their offspring. Offspring and foster infants were matched for age (difference $=$ 0-17 days; mean $=4.9$ ) and sex. The 60 mothers were tested in different postpartum periods, as follows: 11 at age $0-0.5$ months, 11 at $0.5-1$ months, 10 at $1.0-2.0$ months, 9 at 2.0-3.0 months, 6 at 3-4 months, 10 at $4-5$ months, and 3 at 5-6 months. Instead of assessing the outcome of the temporary adoption as successful or unsuccessful, the mother's behavior with her own offspring and with the foster infant was compared. In the first period (0-0.5 postpartum months) there were no differences in maternal behavior. In the second period (0.5-1.0 months), significant differences in lipsmacking and sniffing emerged. Aggression toward foster infants also began during this period and became significantly different after the second month. These findings were interpreted as suggesting that maternal discrimination of offspring occurs gradually, being absent in the first two postpartum weeks and becoming fully established after the second month.

On the basis of the cross-fostering data analyzed in the previous section and some of the studies reviewed in this section, the information provided by the experimental studies of infant adoption can be summarized as follows. In general, the earlier the cross-fostering is attempted after parturition, the higher the probability of successful adoption of a foster infant. Cross-fosterings conducted within 1-2 weeks after parturition have a high probability 
of success whereas those attempted several months after parturition are likely to fail. However, for cross-fosterings conducted within 2-3 weeks after parturition, the time elapsed since parturition is not necessarily a good predictor of adoption success. In addition to time since parturition, the time elapsed since mother and infant were separated and the adoption was attempted appears to be a good predictor of adoption success. Specifically, although some individual studies did not find an effect of separation time on adoption (perhaps because of the use of inaccurate information), the analysis of the crossfostering data set indicates that adoption attempts following a shorter separation are significantly more likely to succeed than those following a longer separation. The combined data set included only one case in which separation time exceeded $24 \mathrm{~h}$. It is likely that if longer separations had been included, the effect of separation time on adoption success would have been even more apparent. Differences in age (at least within the range of a few days) or sex between offspring and foster infant do not appear to significantly affect the probability of adoption. Finally, although some macaque mothers will adopt and raise two foster infants, adoption of twins is far more likely to fail than adoption of a single infant. The birth of twins and the adoption of two infants are rare events in macaques and when they occur, they place a considerable energetic burden to their mothers (e.g., Ellesworth \& Andersen, 1997). Thus, inducing this phenomenon in the laboratory can be successful only under rare and unusual circumstances.

\section{Studies of Mother-Infant Recognition}

One of the earliest studies investigating the mother's ability to discriminate her own offspring was conducted by Jensen (1965) with pigtail macaques. Five individually housed mothers were separated from their infants on several successive sessions starting in the early postpartum days. The test consisted of comparing the mother's locomotor activity in her home cage (pacing) when her own infant or an alien infant was housed in an adjacent cage. Two mothers were tested with their own infant or a 3- to 5-month-old alien infant. Two mothers were tested with their own infant or an alien infant only 4-10 days older than their own. One mother was tested with both an older and a same-aged infant. Mothers reduced pacing when tested with their own infant. From the day of parturition and throughout the first month, 3 mothers gave evidence of discrimination between their own infant and the 3- to 5-month-old infant. Two mothers tested with same-aged infants showed similar responses on the day of parturition and on days 3-4. On days 6-7, they started discriminating and thereafter reacted with indifference to the alien infant. Although the mother's locomotor activity is a rather rough indicator of offspring discrimination, these findings suggest that gross differences in infant age are discriminated from the beginning, whereas young infants of similar age are discriminated within a week. This study, however, provided no information as to whether visual, vocal, or olfactory cues were used for offspring discrimination. 
Evidence that visual cues are not necessary for early offspring recognition was provided by a study of galagos (Galago crassicaudatus and G. senegalensis), a small nocturnal prosimian (Klopfer, 1970). In this study, three female G. crassicaudatus and two female G. senegalensis were tested in an experimental apparatus in which they could choose to spend time near a box containing their biological offspring or an alien infant of the same species, sex, and age. The females were repeatedly tested during their first 5 postpartum weeks under two different conditions: one in which they could see, hear, and smell the infants and the other in which visual cues were not available. This study demonstrated that mothers did not recognize their offspring at 4 days of age but they did so at 7 days and that offspring recognition could occur through auditory and olfactory cues alone.

Most subsequent studies of mother-infant recognition in primates have investigated whether mothers can discriminate their offspring's vocalizations, perhaps because vocal cues can be more easily manipulated than visual or olfactory ones. The earliest studies of mother-infant vocal recognition, however, provided negative results.

Simons, Bobbitt, and Jensen (1968) tested two pigtail macaque mothers for vocal recognition of their 7-month-old female infants by separating the infants and exposing the mothers to the recorded vocalizations of their own infants or of strange infants. No differences in locomotor activity or maternal vocalizations were noted in relation to the vocalizations of their own and the strange infants. In a follow-up study, Simons and Bielert (1973) tested 6 pigtail macaque mothers with their 3- to 4-month-old infants in soundproof rooms equipped with microphones and speakers where they could hear the vocalizations of their own infants or those of strange infants matched for sex and age. Again, there were no significant differences in the mothers' locomotor activity or vocalizations in relation to infant vocalizations. Although these findings seem to suggest that mothers could not vocally recognize their infants, the negative results could be an artifact of the stressful procedures with which the animals were tested. In fact, virtually all of the subsequent studies of mother-infant vocal recognition in macaques provided positive evidence even when mothers were tested with much younger infants. For example, Japanese macaque mothers recognized the calls of their 22to 23-month-old offspring when tested with playback experiments (Pereira, 1986). In Barbary macaques (Macaca sylvanus), mothers vocally recognized their 4- to 8 week-old infants both under naturalistic conditions and with playback experiments (Hammerschmidt \& Fischer, 1998). Finally, in rhesus macaques, there is evidence that mothers can vocally recognize their infants as early as the second postpartum week (Jovanovic, Megna, \& Maestripieri, 2000). Studies of Japanese macaques have demonstrated that infants can recognize their mothers, both visually and vocally, within the first few weeks of life (Masataka, 1985; Nakamichi \& Yoshida, 1986; see also Hansen, 1976, for rhesus macaques). 
Maternal recognition of offspring on the basis of vocal cues has been demonstrated also in squirrel monkeys and vervet monkeys (Cercopithecus aethiops). In one study of squirrel monkeys, mothers responded differentially (mostly through their own vocalizations and proximity) to the vocalizations of their offspring and those of other infants (offspring and control infants ranged in age from 1 to 7 months; Kaplan, Winship-Ball, \& Sim, 1978). These findings were confirmed by a subsequent study showing that squirrel monkey mothers can vocally recognize their 9-month-offspring through both live-infant tests and playback tests (Symmes \& Biben, 1985). Playback tests were also used in a study of vervet monkeys, in which mothers were shown to recognize the vocalizations of their 2-year-old offspring (Cheney \& Seyfarth, 1980).

Although no study has specifically investigated whether maternal recognition can occur shortly after parturition, some observations made in our recent infant cross-fostering study (Maestripieri et al., 2000) suggest that this may be the case. In this study, some of the abusive mothers who failed to adopt a 1 or 2-day-old infant initially approached the infant, sniffed it, and avoided it thereafter, suggesting that they could discriminate the alien infant. One nonabusive mother rejected a foster infant the day after parturition (individual Qw1 in Table 1) but subsequently accepted her own infant after having sniffed it for a few seconds. Finally, in one case where two infants born in the same group were switched $24 \mathrm{~h}$ after birth, the following day the two infants were found to be back with their biological mothers, suggesting that the latter had a clear idea of which infant belonged to which mother. These observations suggest that mothers may be able to recognize their infants as early as $24 \mathrm{~h}$ after birth, probably on the basis of olfactory cues.

In conclusion, although primate mothers appear to be able to discriminate their offspring from much older infants right after parturition, the estimates of the earliest maternal discrimination between offspring and infants of same sex and age range from a few hours after parturition to 3-4 postpartum weeks. Some of the discrepancies among the findings reported by different studies are probably due to the different methodological procedures involved. Although no study has specifically investigated early olfactory recognition of offspring in primates, preliminary observations suggest that mothers can recognize their infant's odor a few hours after birth. This is not surprising considering that in humans, new mothers have been shown to discriminate their newborn's odor as early as a few hours postpartum (Porter, 1991).

\section{IS THE BONDING HYPOTHESIS SUPPORTED BY THE EVIDENCE?}

In this investigation of the possible occurrence of a sensitive period for mother-infant bonding in primates, my working hypothesis has been that a gradual process of selective bonding to one infant begins at parturition and is 
completed at some point during the early postpartum period. In this section, I will discuss whether the information previously presented lends support to the mother-infant bonding hypothesis. Then I will discuss the possible mechanisms underlying mother-infant bonding in primates, and finally I will discuss the potential evolutionary significance of the bonding phenomenon in primates, humans, and other mammals.

Naturalistic observations of infant adoption in primates suggest two main considerations that are relevant to mother-infant bonding. First, the willingness of newly parturient females to adopt an alien infant, particularly if they already have one of their own, suggests that the postpartum period is characterized by enhanced responsiveness to infants. The observation, however, that mothers who lost their offspring often adopt an infant of the same sex and age as the offspring suggests that the postpartum responsiveness to infants is not entirely indiscriminate. Second, although infant mortality is by no means entirely concentrated in the first few days of life, most reported cases of infant adoption occurred within the first 2-3 weeks postpartum. The fact that no cases of successful adoption have been reported beyond this period may not be a coincidence and suggests that the potential for adoption of alien infants is to some extent limited to the immediate postpartum period. Taken together, the naturalistic observations of infant adoption in primates support the first prediction of the bonding hypothesis, that there exists a limited period of time after parturition during which, if mother and infant are separated (as in the case of infant death), the mother will likely adopt an alien infant with similar characteristics.

Two main conclusions can be drawn from the infant cross-fostering studies in primates. One general conclusion is that the earlier the cross-fostering is attempted after parturition, the higher the probability of successful adoption. Cross-fosterings conducted within 1-2 weeks after parturition have a high probability of success, whereas those attempted several weeks or months later are more likely to fail. This finding concurs with the naturalistic observations of infant adoption in suggesting the existence of a postpartum sensitive period during which separated mothers are likely to adopt an alien infant. Studies such as the Jensen and Tolman (1962), in which macaque mothers were briefly separated from their infants at 5-7 months postpartum and reunited with either their offspring or a strange infant, clearly support the third prediction of the bonding hypothesis, stating that when mother and infant are separated after the bonding period and later reunited, the mother will accept her own infant but reject any other infant. Maternal recognition and acceptance of older offspring has also been demonstrated following long separations, e.g., by a study by Gordon et al. (1992) in which rhesus macaque mothers accepted their 2-year-old offspring after an 18-week separation.

The infant cross-fostering data evidence some variability among individual mothers in their tendency to adopt or reject foster infants, so that some mothers may reject a foster infant even in the immediate postpartum period, 
whereas others may successfully adopt one 2 or 3 weeks later. Such variability does not contradict the bonding hypothesis, which accounts for normative aspects of maternal responsiveness during the postpartum period as well as for individual differences in responsiveness (see below).

The second prediction of the bonding hypothesis is that when mother and infant are separated during the sensitive period, the mother will reject her own infant and any other infant if reunion occurs after the end of the sensitive period. Determining exactly the end of the bonding period may be a difficult or impossible task because such period may end gradually instead of abruptly and because its duration may vary among individuals. One testable corollary of the second prediction, however, is that when mother and infant are separated during the bonding period, the probability of infant acceptance should be higher the shorter the time interval since separation. Thus, if the mother is reunited with either her own infant or an alien infant after a relatively long separation, she would be expected to reject both infants.

The prediction that mothers should be more likely to adopt a foster infant after a short separation than after a long separation was supported by the analysis of cross-fostering data. It is noteworthy that separation time was a good predictor of adoption success even if the variability in separation time was in the range of minutes, and not hours or days. With longer separations, the effect of this variable on adoption success would probably be more marked. Unfortunately, no quantitative information was available about separation and reunion between the mothers and their biological offspring. Qualitative observations, however, suggest that when young infants are momentarily separated from their mothers for routine procedures, there is a high probability that they will be accepted by their mothers upon reunion, whereas infants that are separated from their mothers for $24 \mathrm{~h}$ or more are more likely to be rejected (personal observation).

The cross-fostering data indicate that the variability in the outcome of adoption attempts is not accurately accounted for by differences in age or sex between offspring and foster infants. Although these findings may suggest that mothers are willing to adopt newborn infants regardless of their age or sex, it cannot be ruled out that if mothers were given the choice between two foster infants to adopt, they would choose the one that is most similar to their own offspring. In a cross-fostering experiment, however, where mothers do not have such a choice, any infant of either sex and within a certain age range may be better than no infant at all.

Taken together, the cross-fostering studies strongly support the first and the third prediction of the bonding hypothesis. The second prediction of the bonding hypothesis is also supported by the data although a more comprehensive test of this prediction would require a wider range of separation lengths and information on separation and reunion between mother and offspring.

Most studies aimed at investigating whether primate mothers can visually 
or vocally discriminate between their offspring and same-aged infants have provided evidence that offspring recognition takes place within the first 24 weeks after parturition. No study has systematically investigated whether offspring recognition can occur in the first few hours after parturition. Some qualitative observations, however, suggest that that may be the case and that early recognition may be based on olfactory cues. Although the data on mother-offspring recognition are not directly relevant to the testing of the bonding hypothesis, they provide information potentially useful to understanding the mechanisms underlying bonding.

\section{BONDING MECHANISMS}

The information previously reviewed suggests that there exists an initial postpartum period in primates characterized by enhanced maternal responsiveness to infants and propensity to adopt alien infants. It may be argued, however, that the potential for adoption does not necessarily demonstrate the existence of a sensitive period for mother-infant bonding. Instead, it may be argued that the first 2-3 postpartum weeks simply represent a learning period during which mothers gradually become familiar with the olfactory, visual, and vocal characteristics of their infants to the point where they will be able to discriminate their offspring from other infants. In this view, mothers would be initially responsive to any infant and likely to adopt alien infants after infant loss or separation (first prediction of the bonding hypothesis) simply because they cannot discriminate between their own and alien infants. It may also be argued that if mothers are separated from their infants during the first 2-3 postpartum weeks and reunited with them or with alien infants after a long time, the learning process would be disrupted. Such disruption of learning could lead mothers to "forget" that they had an infant in the first place, thus leading to a high probability of rejection (second prediction of the bonding hypothesis). If the learning process is not disrupted, however, mothers will eventually learn to discriminate their offspring and any separation and reunion taking place after discrimination has been achieved would be likely to be followed by acceptance of their own infant and rejection of any other alien infant (third prediction of the bonding hypothesis).

The hypothesis that the bonding period corresponds to the period necessary for mothers to learn to recognize their infants is simple and intuitively appealing. A closer examination of the evidence, however, suggests that this hypothesis does not provide an adequate explanation for the phenomena highlighted by the naturalistic and experimental studies of infant adoption in primates. First of all, some observations previously reported suggest that mothers are capable of recognizing their infants as early as 24-48 h after parturition. This finding argues against the notion that mothers adopt alien infants because they cannot discriminate them from their offspring. If offspring discrimination is achieved within the first 1-2 days postpartum, however, it is unclear why mothers are likely to adopt alien infants in the second or third postpartum week and unlikely to do so thereafter. 
In addition to underestimating the mother's ability for early offspring recognition, the hypothesis that adoption occurs because of lack of offspring discrimination does not fit well with the variability in the outcome of infant cross-fostering. If this hypothesis were correct, one would have to argue that there exists considerable variability among individual mothers in their ability to learn and recognize their infants' characteristics so that the mothers who reject alien infants in the first 2 postpartum weeks are better at this task than the mothers who adopt them. Since individual differences in the propensity to adopt alien infants are found despite similarities in previous experience with infants, one would have to argue that these differences reflect biologically based variability in sensory, perceptual, and memory skills such as those involved in the perception, processing, and retrieval of infant stimuli. The notion that individuals of the same species, who live in the same environment and who are often genetically related to each other, exhibit such variability in cognitive skills is quite implausible. For example, we recently reported that rhesus macaque abusive mothers are more likely to reject alien infants during an infant cross-fostering procedure than nonabusive mothers (Maestripieri et al., 2000). It is quite unlikely, however, that this difference is due to the abusive mothers being better at recognizing their infants than the nonabusive mothers.

Finally, the recognition hypothesis does not explain why mothers with a live infant occasionally adopt an additional infant, unless one is willing to argue not only that mothers cannot discriminate between their own and an alien infant but also that they cannot discriminate between having one or two infants. Thus, from the perspective of the recognition hypothesis, adoption of an alien infant following infant loss and adoption of an additional infant are different phenomena dependent on different proximate mechanisms.

An alternative hypothesis that can account for the findings of naturalistic and experimental studies of infant adoption is that what characterizes the early postpartum period is not a gradual process of offspring recognition but the particular motivational state of the mother. In this view, the first postpartum weeks are characterized by a state of heightened motivation to interact with infants, which gradually decreases over time. Thus the first postpartum weeks are a true maternal sensitive period in the sense that mothers are more sensitive and responsive to infant stimuli during this period than during subsequent periods. Although this period is characterized by high motivation to interact with infants in general, under normal circumstances such high maternal motivation is channeled into interactions with the offspring. Therefore, the sensitive period is also a period characterized by the formation of a strong bond between mother and offspring. This does not imply, however, that if the opportunity to form a strong bond with the infant during this period is missed, such bond cannot be formed at a later stage.

The motivational hypothesis accounts for the three predictions of the bonding hypothesis as follows. First, mothers display a high propensity to adopt alien infants during the sensitive period not because they do not dis- 
criminate their offspring but because they are highly motivated to interact with an infant; thus, after infant death or separation, an alien infant is better than no infant at all. Second, mothers who are separated from their offspring during the sensitive period and reunited with them or with an alien infant after a long time are likely to reject any infants not because a learning process has been disrupted but because a long separation from the infant may be associated with a reduction in maternal motivation. Such a reduced maternal motivation could be due to neuroendocrine changes associated with a long separation from the infant (see below), including perhaps return to estrous. Third, if separation and reunion occur after the end of the bonding period, mothers are likely to accept their offspring and reject alien infants not because they have achieved offspring recognition but because their maternal motivation is not strong enough to support the adoption of an alien infant.

In addition to providing a plausible account for the bonding phenomena, the hypothesis that the sensitive period has a strong motivational basis also accounts for individual differences in the propensity to adopt infants better than the recognition hypothesis. In fact, it is more plausible to postulate individual differences in maternal motivation than individual differences in sensory abilities or cognitive processes. For example, it is more plausible to argue that abusive mothers are more likely to reject foster infants because they have lower maternal motivation than because they are better at recognizing their offspring (Maestripieri et al., 2000). Finally, from the perspective of the motivational hypothesis, adoption of an alien infant after infant loss and adoption of an additional infant are two aspects of the same phenomenon and do not require independent explanations. Thus, the motivational hypothesis possesses the added advantage of parsimony relative to the recognition hypothesis because it can account for two different phenomena by invoking only a single parameter.

Similar to other mammals, heightened maternal motivation after parturition may be dependent on the neuroendocrine changes accompanying late pregnancy, parturition, and lactation (Maestripieri, 1999; in press). For example, there is evidence in macaques that the high levels of circulating estradiol prior to parturition are responsible for the increased maternal responsiveness observed in this period (Maestripieri \& Zehr, 1998). Moreover, in common marmosets, treatment with estrogen and progesterone in concentrations similar to those of late pregnancy significantly increased female responsiveness to infants (Pryce, Dobeli, \& Martin, 1993). The hormonal concentrations of late pregnancy could also affect postpartum maternal motivation. In a species of New World monkeys, the red-bellied tamarin (Saguinus labiatus), Pryce, Abbott, Hodges, and Martin (1988) reported that mothers with more intense levels of postpartum care and whose infants survived had higher urinary concentrations of estradiol in the last week of pregnancy than mothers whose infants did not survive. In humans, mothers who maintained high levels of estradiol over the parturitional period also had higher feelings 
of attachment to their own infant in the early postpartum days than mothers with lower levels of estradiol (Fleming et al., 1997).

In addition to the late pregnancy hormones, postpartum maternal motivation could also be supported by the neuroendocrine changes accompanying lactation, particularly the release of hormones and neurotransmitters such as oxytocin, prolactin, vasopressin, and endogenous opioids (Carter, 1998). Since these substances are known to affect maternal motivation in some mammals (Numan, 1994) and perhaps also in humans (Uvnas-Moberg, Windstrom, Nissen, \& Bjorvell, 1990; Uvnas-Moberg, 1998), it is possible that reduced maternal motivation associated with a long separation from the infant during the early postpartum period could be mediated by altered secretion of hormones such as oxytocin or prolactin. These hypotheses concerning biological influences on maternal motivation in the postpartum period are clearly speculative but recent studies suggest that primate and human parenting have more in common with the maternal behavior of other mammals than previously thought (Maestripieri, 1999, in press).

\section{EVOLUTIONARY CONSIDERATIONS AND IMPLICATIONS FOR HUMAN RESEARCH}

In their review of the literature on mother-infant bonding in animals and humans, Lamb and Hwang (1982) argued that among mammals, only in ungulates does there exist a narrow sensitive period during which the mother learns the characteristics of her own young so that she can thereafter respond selectively to it. They argued that the occurrence of mother-offspring bonding in ungulates can be accounted for by the extreme precocity of the young and the social ecology of ungulate groups. In ungulates, the young are able to walk independently and follow their mothers within minutes after birth, and because these animals usually travel in large herds, there is considerable risk that mothers may accidentally swap their young. Therefore, it would seem adaptive for ungulate mothers to recognize their offspring and bond with them quickly after birth. Lamb and Hwang (1982) argued that primates and humans do not share any of the characteristics of the species in which there occurs a sensitive period for mother-infant bonding. Human infants are so incompetent motorically that pressure to incorporate a rapid process of maternal recognition and bonding is unlikely to have occurred during our evolution. Thus, they concluded that there is no reason to believe that a brief sensitive period would have been adaptive in humans.

The primate data reviewed in this article suggest that mothers recognize their infants quite early after parturition. Even though olfaction is underdeveloped in primates and humans relative to other mammals such as rodents or carnivores, monkey and human mothers are probably capable of recognizing their infant's odor within hours after birth and learn their visual and vocal characteristics shortly afterward. Whether early offspring recognition is an ability that evolved in response to specific selection pressures, such as the 
risk of losing the young, or is simply a by-product of primate and human sensory and perceptive skills remains to be assessed.

What I propose in this article is that the immediate postpartum period may be a sensitive period for bonding in primates as in ungulates not because of offspring recognition but because of the new mothers' heightened motivation to interact with infants and propensity to display caregiving behavior. Just because primate infants are, in many respects, so dependent on their mothers, it is crucial not so much that their mothers recognize them, as that they are motivated to take care of them. I would argue then, that there has been strong selective pressure for heightened maternal motivation in the immediate postpartum period to the point that primate mothers are sometimes prone to making evolutionarily maladaptive choices such as adopting an unrelated infant. The circumstances leading to the adoption of an unrelated infant, however, are relatively rare in the natural environment. Under normal circumstances, enhanced maternal motivation during the postpartum period coupled with early offspring recognition ensures that mothers will selectively channel their parental efforts into their biological offspring.

Individual differences in maternal motivation during the postpartum period may also be a product of evolution and have an adaptive value (Daly \& Wilson, 1995). Evolutionary theory predicts that parents should invest in their offspring only when the benefits of parental care outweigh its costs (Trivers, 1972). When circumstances are unfavorable for parental investment, mothers should reduce or terminate parental care as early as possible after parturition. For example, it may be adaptive for very young monkey mothers to abandon their offspring shortly after parturition rather than attempt to raise an infant that is unlikely to survive and risk compromising their own survival and future reproduction (Fairbanks \& McGuire, 1995; Maestripieri \& Carroll, 1998). Thus, there may be marked individual differences in maternal motivation during the postpartum period and such differences could be mediated by hormonal mechanisms.

Some of the phenomena that have been used to argue for bonding in ungulates have been observed in primates as well. For example, similar to ungulates, monkey mothers can be induced to adopt alien infants if these are exchanged with their offspring quickly after birth. Furthermore, similar to ungulates, monkey mothers sometimes reject their own offspring or any other infant if they are separated from their offspring after birth and not reunited with them for a relatively long time. Obviously we should not expect to find exactly the same mother-infant bonding phenomenon in ungulates and primates just as we should not expect any behavioral phenomenon to be exactly the same in animals that are as phylogenetically distant and ecologically different as ungulates and primates. For example, it is very likely that the sensitive period for mother-infant bonding is much shorter in ungulates than in primates for the developmental and ecological reasons discussed by Lamb and Hwang (1982). The mechanisms and processes underlying 
mother-infant bonding, however, may be fundamentally similar in ungulates and primates.

The extent to which mother-infant bonding occurs in humans remains unclear because the type of experimental manipulations performed to assess maternal sensitive periods in ungulates and primates have not been conducted in humans. However, since many aspects of the neuroendocrine regulation of pregnancy, parturition, and lactation are very similar across all mammalian species (Numan, 1994), it is not implausible to hypothesize that mother-infant bonding occurs in humans as well. For example, it is plausible to argue that the immediate postpartum period is a sensitive period for bonding in humans because it is a period characterized by heightened maternal motivation. In fact, recent studies conducted by Fleming and collaborators have provided evidence that some aspects of maternal responsiveness and behavior in the first postpartum days, including the mother's ability to recognize her newborn's odor, are influenced by periparturitional concentrations of hormones such as estrogen and cortisol (Fleming et al., 1997; Fleming, Steiner \& Corter, 1997). It must be emphasized that the fact that the first postpartum weeks may be a sensitive period for maternal motivation does not imply that bonding cannot take place outside this period. The fact that fathers or adoptive mothers can be perfect parents clearly demonstrates that in humans, just like in other mammals, pregnancy, parturition or lactation are not necessary prerequisites for good parenting.

When human research is viewed from a comparative perspective, two main issues are worth emphasizing. First, it is important to note that the type of evidence required to demonstrate the occurrence of mother-infant bonding depends on how this phenomenon is defined. In animals such as ungulates and primates, the bonding period has been characterized as a sensitive period during which mothers show a heightened responsiveness to young (as expressed, for example, in their tendency to adopt alien young), the disruption of which may, under certain circumstances, lead to offspring rejection. In humans, however, mother-infant bonding has mostly been conceptualized in terms of the consequences that different amounts of contact can have for the mother and the child. The failure to unequivocally demonstrate such effects has been interpreted as evidence against the sensitive period. If a broader definition of bonding is used for humans, however, the failure to detect the long-term consequences of small differences in postpartum contact may question some aspects of the bonding phenomenon but not the phenomenon in its entirety.

The second issue to be emphasized is that the working hypothesis adopted in this study predicts that a mother-infant separation occurring during the sensitive period should result in infant rejection only if the reunion with the offspring or the adoption attempt occurs after the end of the sensitive period (i. e., after a significant reduction in maternal motivation has occurred). If the offspring is reunited with the mother before the end of the bonding period, no 
consequences of separation are necessarily expected. Thus, it is possible that the failure of some human studies to report any consequences of early mother-infant separation is due to the short duration of the separation experience.

Ultimately, the assessment of mother-infant bonding in humans depends on how this phenomenon is defined and which of its features are believed to be relevant enough to human parenting and child development to warrant investigation. However, since the idea of bonding and the notion of sensitive period have been imported from animal research, the use of a comparative perspective when conducting research with humans, at both the mechanistic and evolutionary levels of analysis, has both informative and heuristic value. The data obtained with primates could be particularly valuable in this regard because of the many similarities in parenting and developmental processes in primates and humans. In both primates and humans, there exists considerable interindividual variability in maternal motivation during the postpartum period, and this variability also includes pathological expressions (e.g. Kumar, 1997; Maestripieri et al., 2000). The determinants of these individual differences in motivation and their consequences for later parenting and child development are still poorly understood. Primate research in this area can stimulate and complement human research and enhance our understanding of some adaptive and maladaptive processes underlying parenting behavior and child development.

\section{REFERENCES}

Bateson, P. (1966). The characteristics and context of imprinting. Biological Reviews, 41, $177-220$.

Bateson, P. (1979). How do sensitive periods arise and what are they for? Animal Behaviour, 27, 470-486.

Bernstein, I. S. (1991). The correlation between kinship and behaviour in non-human primates. In P. G. Hepper (Ed.), Kin recognition (pp. 6-29). Cambrigde: Cambridge University Press.

Box, H. O. (1984). Primate behaviour and social ecology. London: Chapman and Hall.

Carter, C. S. (1998). Neuroendocrine perspectives on social attachment and love. Psychoneuroendocrinology, 23, 779-818.

Chamove, A. S., \& Anderson, J. R. (1982). Hand-rearing infant stumptailed macaques. Zoo Biology, 1, 323-331.

Champoux, M., Boyce, W. T., \& Suomi, S. J. (1995). Biobehavioral comparisons between adopted and nonadopted rhesus monkey infants. Developmental and Behavioral Pediatrics, 16, 6-13.

Cheney, D. L., \& Seyfarth, R. M. (1980). Vocal recognition in free-ranging vervet monkeys. Animal Behaviour, 28, 362-367.

Cho, F., Suzuki, M., \& Honjo, S. (1986). Adoption success under single-cage conditions by cynomolgus macaque mothers (Macaca fascicularis). American Journal of Primatology, 10, 119-124.

Clarke, M. R., \& Glander, K. E. (1981). Adoption of infant howling monkeys (Alouatta palliata). American Journal of Primatology, 1, 469-472. 
Colgan, P. (1983). Comparative social recognition. New York: Wiley.

Collias, N. (1956). The analysis of socialization in sheep and goats. Ecology, 37, 228-239.

Daly, M., \& Wilson, M. (1995). Discriminative parental solicitude and the relevance of evolutionary models to the analysis of motivational systems. In M. S. Gazzaniga (Ed.), The cognitive neurosciences (pp. 1269-1286). Cambridge, MA: MIT Press.

Deets, A. C., \& Harlow, H. F. (1974). Adoption of single and multiple infants by rhesus monkey mothers. Primates, 15, 193-203.

Ellesworth, J. A., \& Andersen, C. (1997). Adoption by captive parturient rhesus macaques: Biological vs. adopted infants and the cost of being a "twin" and rearing "twins." American Journal of Primatology, 43, 259-264.

Eveleigh, J. R., \& Hudson, C. E. (1973). Successful fostering of a newly born squirrel monkey (Saimiri sciureus). Laboratory Primate Newsletter, 12, 13-14.

Eyer, D. E. (1994). Mother-infant bonding: A scientific fiction. Human Nature, 5, 69-94.

Fairbanks, L. A., \& McGuire, M. T. (1995). Maternal condition and the quality of maternal care in vervet monkeys. Behaviour, 132, 733-754.

Fleming, A. S., Ruble, D., Krieger, H., \& Wong, P. Y. (1997). Hormonal and experiential correlates of maternal responsiveness during pregnancy and the puerperium in human mothers. Hormones and Behavior, 31, 145-158.

Fleming, A. S., Steiner, M., \& Corter, C. (1997). Cortisol, hedonics, and maternal responsiveness in human mothers. Hormones and Behavior, 32, 85-98.

Fuccillo, R., Scucchi, S., Troisi, A., \& D'Amato, F. R. (1983). Newborn adoption in a confined group of Japanese macaques. American Journal of Primatology, 5, 257-260.

Goldberg, S. (1983). Parent-infant bonding: Another look. Child Development, 54, 13551382.

Gordon, T. P., Gust, D. A., Wilson, M. E., Ahmed-Ansari, A., Brodie, A. R., \& McClure, H. M. (1992). Social separation and reunion affects immune system in juvenile rhesus monkeys. Physiology \& Behavior, 51, 467-472.

Gubernick, D. J. (1980). Maternal “imprinting"' or maternal "labelling”' in goats? Animal Behaviour, 28, 124-129.

Hammerschmidt, K., \& Fischer, J. (1998). Maternal discrimination of offspring vocalizations in Barbary macaques (Macaca sylvanus). Primates, 39, 231-236.

Hansen, E. W. (1976). Selective responding by recently separated juvenile rhesus monkeys to the calls of their mothers. Developmental Psychobiology, 9, 83-88.

Hepper, P. G. (Ed.) (1991). Kin recognition. Cambridge: Cambridge University Press.

Hersher, L., Moore, A. U., \& Richmond, J. B. (1958). Effect of postpartum separation of mother and kid on maternal care in the domestic goat. Science, 128, 1342-1343.

Hersher, L., Richmond, J. B., \& Moore, A. U. (1963). Modifiability of the critical period for the development of maternal behavior in sheep and goats. Behaviour, 20, 311-320.

Holman, S. D., \& Goy, R. W. (1988). Responses of foster-mothers and troop members to adopted newborns in a captive group of rhesus monkeys. Primates, 29, 343-352.

Jensen, G. D. (1965). Mother-infant relationship in the monkey Macaca nemestrina: Development of specificity of maternal response to own infant. Journal of Comparative and Physiological Psychology, 59, 305-308.

Jensen, G. D., \& Tolman, C. W. (1962). Mother-infant relationship in the monkey Macaca nemestrina: The effect of brief separation on mother-infant specificity. Journal of Comparative and Physiological Psychology, 55, 131-136.

Jovanovic, T., Megna, N. L. \& Maestripieri, D. (2000). Early maternal recognition of offspring vocalizations in rhesus macaques (Macaca mulatta). Primates, 41, 421-428. 
Kaplan, J., Winship-Ball, A., \& Sim, L. (1978). Maternal discrimination of infant vocalizations in squirrel monkeys. Primates, 19, 187-193.

Kennell, J. H., \& Klaus, M. H. (1984). Mother-infant bonding: Weighing the evidence. Developmental Review, 4, 275-282.

Keverne, E. B., \& Kendrick, K. M. (1990). Neurochemical changes accompanying parturition and their significance for maternal behavior. In N. A. Krasnegor \& R. S. Bridges (Eds.), Mammalian parenting (pp. 281-304). Oxford: Oxford University Press.

Klaus, M. H., \& Kennell, J. H. (1976). Maternal-infant bonding. St. Louis, MO: Mosby.

Klaus, M. H., \& Kennell, J. H. (1982). Parent-infant Bonding. St. Louis, MO: Mosby.

Klopfer, P. H. (1970). Discrimination of young in galagos. Folia Primatologica, 13, 137143.

Klopfer, P. H. (1971). Mother love: What turns it on? American Scientist, 59, 404-407.

Klopfer, P. H., Adams, D. K., \& Klopfer, M. S. (1964). Maternal imprinting in goats. Proceedings of the National Academy of Science U.S.A., 52, 911-914.

Kumar, R. C. (1997). “Anybody's child'”: Severe disorders of mother-to-infant bonding. British Journal of Psychiatry, 171, 175-181.

Lamb, M. E., \& Hwang, C. P. (1982). Maternal attachment and mother-neonate bonding: A critical review. In M. E. Lamb \& A. L. Brown (Eds.), Advances in developmental psychology (pp. 1-39). Hillsdale, NJ: Erlbaum.

Lawrence, W. A. (1982). Bilateral infant transfer and adoption in olive baboons (Papio cynocephalus). Journal of Human Evolution, 11, 505-510.

Lorenz, K. (1937). The companion in the bird's world. Auk, 54, 245-273.

Maestripieri, D. (1999). The biology of human parenting: Insights from nonhuman primates. Neuroscience and Biobehavioral Reviews, 23, 411-422.

Maestripieri, D. Biological bases of maternal attachment. Current Directions in Psychological Science, in press.

Maestripieri, D., \& Call, J. (1996). Mother-infant communication in primates. Advances in the Study of Behavior, 25, 613-642.

Maestripieri, D., \& Carroll, K. A. (1998). Child abuse and neglect: Usefulness of the animal data. Psychological Bulletin, 123, 211-223.

Maestripieri, D., Megna, N. L., \& Jovanovic, T. (2000). Adoption and maltreatment of foster infants by rhesus macaque abusive mothers. Developmental Science, 3, 287-293.

Maestripieri, D., \& Wallen, K. (1995). Interest in infants varies with reproductive condition in group-living female pigtail macaques (Macaca nemestrina). Physiology and Behavior, 57, 353-358.

Maestripieri, D., \& Zehr, J. L. (1998). Maternal responsiveness increases during pregnancy and after estrogen treatment in macaques. Hormones and Behavior, 34, 223-230.

Marsden, H. M., \& Vessey, S. H. (1968). Adoption of an infant green monkey within a social group. Communications in Behavioral Biology, 2, 275-279.

Masataka, N. (1985). Development of vocal recognition of mothers in infant Japanese macaques. Developmental Psychobiology, 18, 107-114.

Meier, G. W. (1965). Maternal behaviour of feral- and laboratory-reared monkeys following the surgical delivery of their infants. Nature, 206, 492-493.

Myers, B. J. (1984). Mother-infant bonding: The status of the critical period hypothesis. Developmental Review, 4, 240-274.

Nakamichi, M., \& Yoshida, A. (1986). Discrimination of mother by infant among Japanese macaques (Macaca fuscata). International Journal of Primatology, 7, 481-489. 
Nakamichi, M. (1998). Relationships between foster mothers and adopted infants and motherinfant separation in gorillas at San Diego Wild Animal Park. Primate Research, 14, 263.

Negayama, K., \& Honjo, S. (1986). An experimental study on developmental changes of maternal discrimination of infants in crab-eating monkeys (Macaca fascicularis). Developmental Psychobiology, 19, 49-56.

Numan, M. (1994). Maternal behavior. In E. Knobil \& J. D. Neill (Eds.), The physiology of reproduction (2nd ed., pp. 221-302). New York: Raven Press.

Ogawa, H. (1998). Adoption and social interactions between a mother and 'twin' offspring in Macaca fuscata. Folia Primatologica, 69, 100-105.

Owren, M. J., \& Dieter, J. A. (1989). Infant cross-fostering between Japanese (Macaca fuscata) and rhesus macaques (M. mulatta). American Journal of Primatology, 18, 245-250.

Pereira, M. (1986). Maternal recognition of juvenile offspring coo vocalizations in Japanese macaques. Animal Behaviour, 34, 935-937.

Porter, R. H. (1991). Mutual mother-infant recognition in humans. In P. G. Hepper (Ed.), Kin recognition (pp. 413-432). Cambridge: Cambridge University Press.

Pryce, C. R., Abbott, D. H., Hodges, J. H., \& Martin, R. D. (1988). Maternal behavior is related to prepartum urinary estradiol levels in red-bellied tamarin monkeys. Physiology and Behavior, 44, 717-726.

Pryce, C. R., Dobeli, M., \& Martin, R. D. (1993). Effects of sex steroids on maternal motivation in the common marmoset (Callithrix jacchus): Development and application of an operant system with maternal reinforcement. Journal of Comparative Psychology, 107, 99-115.

Salzen, E. A., \& Meyer, C. C. (1968). Reversibility of imprinting. Journal of Comparative and Physiological Psychology, 66, 269-275.

Schino, G., Aureli, F., D’Amato, F. R., D’Antoni, M., Pandolfi, N., \& Troisi, A. (1993). Infant kidnapping and co-mothering in Japanese macaques. American Journal of Primatology, 30, 257-262.

Siegel, E. (1982). Early and extended maternal-infant contact: A critical review. American Journal of Diseases of Childhood, 136, 251-257.

Simons, R. C., \& Bielert, C. F. (1973). An experimental study of vocal communicaton between mother and infant monkeys (Macaca nemestrina). American Journal of Physical Anthropology, 38, 455-461.

Simons, R. C., Bobbitt, R. A., \& Jensen, G. D. (1968). Mother monkeys' (Macaca nemestrina) responses to infant vocalizations. Perceptual and Motor Skills, 27, 3-10.

Sluckin, H. M., \& Sluckin, W. A. (1982). Mother-to-infant 'bonding', Journal of Child Psychology \& Psychiatry, 23, 205-221.

Smith, S. (1986). Infant cross-fostering in rhesus monkeys (Macaca mulatta): A procedure for the long-term management of captive populations. American Journal of Primatology, 11, 229-237.

Symmes, D., \& Biben, M. (1985). Maternal recognition of individual infant squirrel monkeys from isolation call playbacks. American Journal of Primatology, 9, 39-46.

Taub, D. M., Lehner, N. D. M., \& Adams, M. R. (1971). Enforced adoption and successful raising of a neonate squirrel monkey Saimiri sciureus. Laboratory Primate Newsletter, 16, $8-10$.

Thierry, B., \& Anderson, J. R. (1986). Adoption in anthropoid primates. International Journal of Primatology, 7, 191-216.

Timmermans, P. J. A., Schouten, W. G. P., \& Krijnen, J. C. M. (1981). Reproduction of cynomolgus monkeys (Macaca fascicularis) in harems. Laboratory Animals, 15, 119123. 
Trivers, R. L. (1972). Parental investment and sexual selection. In B. Campbell (Ed.), Sexual selection and the descent of man (pp. 136-179). Chicago: Aldine.

Uvnas-Moberg, K. (1998). Oxytocin may mediate the benefits of positive social interaction and emotions. Psychoneuroendocrinology, 23, 819-835.

Uvnas-Moberg, K., Windstrom, A. M., Nissen, E., \& Bjorvell, H. (1990). Personality traits in women 4 days postpartum and their correlation with plasma levels of oxytocin and prolactin. Journal of Psychosomatic Obstetrics and Gynaecology, 11, 261-272.

van Wulfften Palthe, T., \& van Hooff, J. A. R. A. M. (1975). A case of adoption of an infant chimpanzee by a suckling foster chimpanzee. Primates, 16, 231-234.

Received: November 22, 1999; revised: April 30, 2000 